\title{
Joint European XFEL and DESY Photon Science Users' Meeting 2015
}

The European XFEL and DESY Photon Science Users' Meeting 2015 broke the attendance record of the previous year. In total, over 800 scientists from around the world came to Deutsches ElektronenSynchrotron (DESY) in Hamburg, Germany, to participate in this three-day event, which took place from 28-30 January 2015. In particular, the latest news about the construction of the European XFEL facility as well as the extension projects at DESY's synchrotron source PETRA III and the Free-Electron Laser FLASH, attracted a lot of interest.

\section{European XFEL}

More than 600 participants attended the first day's sessions, which focused on the European XFEL. The DESY auditorium was packed with scientists eager to hear about the progress of construction and installation. European XFEL Managing Director Massimo Altarelli presented the facility's current status. All six scientific instruments are expected to provide a total of 1000 hours to early user experiments in 2017, with full simultaneous operation of all instruments and over 2000 hours of user operation in 2018, increasing to 4000 by 2019.

Most of the civil construction work for the European XFEL facility has been finished, with the exception of the headquarters (XHQ), the laboratory and office building being erected on top of the experiment hall, which will house the six scientific instruments.

Mass production is in full swing in all areas of the project, and halls on the DESY site and elsewhere are filling with components waiting to be installed in the 5.8-km-long tunnels, shafts, and halls. Technical infrastructure is being installed and the first accelerator modules have been mounted to the roof of the accelerator tunnel.

All 91 undulator sections (each $5 \mathrm{~m}$ long) have been produced, shipped to Hamburg, and tested in dedicated magnetic laboratories for final parameter adjustments. A myriad of components needed for the many-km-long electron beamline sections are being shipped from the members of the international Accelerator Consortium led by DESY.

The European XFEL Accelerator Consortium coordinator, DESY scientist Hans Weise, reported on the continuing assembly and installation work on the 100 accelerator modules and additional components. The facility's electron injector will begin generating electron bunches this summer (electron bunches were produced and accelerated by the RF gun in February).

Other talks detailed the specific electron beam parameters of the first user operation run, the Karabo control and data analysis framework, the beamline installations in the photon tunnels, and the continuing research and design work on the six initial scientific instruments.

Similar to the past few years, 42 students from 17 countries including Italy, Russia, Slovakia and Turkey, received European XFEL travel fellowships to come to the Users' Meeting.

The Thursday events focused on soft X-ray experiments at free-electron lasers (FELs). The morning session was dedicated to the status and recent developments of FLASH at DESY, the unique vacuum ultraviolet (VUV) and soft X-ray FEL, routinely operated for users for the past 10 years.

One highlight was the presentation on first lasing of the second FEL line at FLASH. 
The mounting of all components, a new electron beamline, and 12 tunable undulator modules was completed in January 2014. First lasing was achieved in August 2014 by the new FEL line, FLASH2, at DESY during parallel operation of the existing FLASH1 line. FLASH1 routinely delivers FEL radiation up to $300 \mathrm{eV}(4 \mathrm{~nm})$ for user experiments with pulse durations of 50-200 fs (FWHM). A recent upgrade of the RF system has increased the timing stability of FLASH1 to below $30 \mathrm{fs}$ ( $\mathrm{rms}$ ). The variable gap undulators of FLASH2 allow fast tuning of the wavelength without changing the accelerator parameters. An energy range of $30-225 \mathrm{eV}(5.5-42 \mathrm{~nm})$ has been demonstrated at FLASH2 so far; however, the accessible parameter range is not yet fully explored.

Benefitting from the simultaneous-and almost independent-operation of these two FEL lines, the capacity of beamtime for FLASH users will almost double after the commissioning time at the end of 2015. The new FLASH experiment hall will be able to host up to six new beamlines; one part of this year's poster session at the Users' Meeting-took place there.

Upgrades to the existing FLASH beamlines have been carried out as well. Two permanent end stations are now in operation: the Raman spectrometer on PG1, a two-stage, high-resolution XUV grating spectrometer for inelastic scattering and the "CAMP" (Chamber for Atomic and Molecular Physics) station on BL1. This experiment chamber, which was developed by the Max Planck Advanced Study Group at the Center for Free-Electron Laser Science (CFEL) at DESY and successfully operated at LCLS, is a multi-purpose instrument for electron and ion spectroscopy, pump-probe, and imaging experiments.

The entire morning sessions gave a good overview of the research capabilities at FELs. Magnetization dynamics and time-resolved electron spectroscopy were discussed in subsequent talks, followed by ultrafast dynamics in solids, clusters, and molecules as well as recent results obtained at the "low density matter" (LDM) beamline at the Free Electron Laser for Multidisciplinary Investigations (FERMI) at Elettra Sincrotrone Trieste. These talks demonstrated the huge potential of FEL studies to follow ultrafast processes, e.g. rearrangement of charges in molecules and solids.

\section{Satellite meetings and workshops}

On Thursday afternoon, several satellite meetings and workshops took place.

\section{Meeting on EXAFS Beamlines P64 and P65 (about 45 participants)}

EXAFS Beamlines P64 and P65, part of the PETRA III extension project, will become operational in autumn 2015. Several talks informed the prospective users about the actual status of the two beamlines and the instrumentation projects undertaken by external groups. The workshop concluded with a discussion about the conditions for first user experiments and possible collaborations in the first experiments after the early commissioning phase.

\section{High Energy X-Ray Scattering Workshop (about 60 participants)}

Current and future users, in particular from the German and Swedish user community, participated in this workshop. Beamline scientists working with high energy X-rays at PETRA III gave an overview of the newest developments and possibilities at the existing and future beamlines (P07, P02.1, P21.1, and P21.2) operated by DESY. Moreover, users reported on recent results and future needs. The talks were related to materials science, chemistry, and surface science as well as detector developments.

\section{SAXS/WAXS/GISAXS-User Workshop (about 55 participants)}

The SAXS/WAXS/GISAXS-User Workshop addressed the user community of PETRA III Beamline P03 with its micro-focus $X$-ray scattering instrument and the nano-focus end station. The participants presented their recent and planned experiments and discussed possible collaborations and future instrumental developments. 
Workshop on Data Analysis for Serial Crystallography Experiments using FELs and Synchrotrons (about 38 participants)

"Serial crystallography" is the name that has been given to crystallographic experiments in which only one diffraction pattern, or a very small number of patterns, are acquired from each crystal. This workshop concerned methods for analysing data obtained by this technique. Several analysis software programs were described and briefly demonstrated.

\section{Status of the German Engineering Materials Science Centre (GEMS), Helmholtz-Zentrum Geesthacht (HZG) (about 50 participants)}

This year's workshop was dedicated to the great variety of in situ experiments in materials science. Six GEMS users presented their results from PETRA III Beamlines P07 and P03 (Nanofocus Hutch), where novel samples environments with ever increasing complexity are being used. A highlight was certainly the presentation of in situ laser beam welding results.

\section{Workshop on X-Ray Nano-Imaging of Biological and Chemical Systems at PETRA III (about 120 participants)}

This workshop is part of the activities of the Helmholtz Virtual Institute "Nanoscale Imaging of Biological and Chemical Processes". The workshop included an overview of the nano-imaging PETRA III Beamlines P06, P10, and P11 and of user research activities in various areas, such as cellular and tissue imaging in biology, in-situ imaging of aging catalysts, and structure determination of semiconductor nanowires.

\section{Meeting on the HED Scientific Instrument of the European XFEL (about 85 participants)} The meeting provided the user community with an update on the status of design and planned instrumentation of the High-Energy Density Science (HED) instrument. A specific topic was the actual status of experiment chamber and detector integration. In addition, the current status of the split and delay unit was shown. The meeting was followed by a general assembly of the Helmholtz International Beamline for Extreme Fields (HIBEF) User Consortium, which intends to bring additional instrumentation to the HED instrument enabling high energy laser and pulsed magnetic field experiments

\section{Workshop on the FXE Scientific Instrument of the European XFEL (about 40 participants)} The workshop started with a presentation of the Femtosecond X-Ray Experiments (FXE) instrument and its capabilities for time-resolved $X$-ray spectroscopies and the scattering of liquid systems permitting studies of solar energy converters and photocatalytic devices for future energy storage applications. The main scope of this workshop discussed the possibility of including instrumentation for solid samples, and researchers presented examples for the scientific case. The workshop participants agreed to create a network with the aim of compiling the science case in order to identify key requirements for the new instrumentation, which should lead to joint activities for the investigation of solid samples.

\section{Workshop on Photon Beam Diagnostics for the European XFEL (about 80 participants)}

The workshop covered a broad range of diagnostics methods and instrumentation, with several presentations about temporal diagnostics, polarization and wavefront monitoring, spectral diagnostics, seeding laser systems, switching mirrors, and diagnostics developments at the European XFEL, the SPring-8 Angstrom Compact Free Electron Laser (SACLA), and the Paul Scherrer Institute (PSI) SwissFEL facilities.

Satellite Meeting on Experiments at the MID Scientific Instrument of the European XFEL This satellite meeting took place earlier in the week (26-27 January) and focused on the expected first science experiments at the Materials Imaging and Dynamics (MID) instrument, one of the six initial scientific instruments at the European XFEL that will be operational in 2017. This hard X-ray 
instrument will be mainly used for materials science research, but will also be while also being capable of taking "molecular movies" of processes such as catalytic reactions using the European XFEL's repetition rate of 27000 flashes per second.

\section{DESY - PETRA III}

The third day was reserved for the traditional DESY Photon Science Users' Meeting, featuring reports from PETRA III and the extension project and including reports from committees of the user community (DPS-UC, KFS, and ESUO).

The latest news from the PETRA III extension project, which progressed significantly during the last year, was presented by Edgar Weckert, the director in charge of DESY photon science. PETRA III was shut down in February 2014, when the civil engineering work for the two new experiment halls started. These halls are located at the eastern and northern side of the existing experimental hall "Max von Laue" and were completed according to plan by the end of 2014. Two PETRA III tunnel sections had to be rebuilt in parallel to allow for the installation of the machine and components for 11 new beamlines. The regular operation of all PETRA III beamlines in the experimental hall "Max von Laue" is scheduled to start anew in April 2015. As expected, the demand for beamtime after the shutdown was very high.

Other construction work on the DESY campus-for example, on the Centre for Structural Systems Biology (CSSB) building, in the immediate neighbourhood of the PETRA III experiment halls-also progressed very well. The field of structural biology and infection research will benefit from this new centre, which combines multidisciplinary and long-standing expertise of nine partners.

The DESY NanoLab, already partially available for DESY photon science users and in-house scientists, will complement the attractive offers on the DESY site. The installation of "made-to-measure" equipment for structural and chemical characterization on the nano scale made good progress and will develop further during the year 2015. The construction work for a new photon science building housing the new NanoLab facilities will start in autumn 2015.

The exciting science talks on sustainable materials for possible future applications inspired the audience in the main morning session: strong cellulose filaments suitable for wind turbines, flexible polymer tandem solar cells, in situ surface crystallography, and integrative structural biology.

On Friday afternoon, the European XFEL and DESY Photon Science Users' Meeting featured more than 340 posters at two venues, giving an impressive overview of the growing field of photon science research in structural biology, materials science, chemistry, solid state physics, and many other disciplines. The session included an industrial exhibition with 49 vendors for specialized equipment.

Attendees expressed excitement at learning about new facility details, in terms of planning for first or new user experiments, instrumentation, and unique experimental parameters as well as future prospects for science.

Authors: Wiebke Laasch (DESY) and Andreas Schwarz (European XFEL), Hamburg, Germany

\section{Acknowledgement}

This article was prepared with the help of Karen Appel (European XFEL), Carsten Bähtz (HZDR), Christian Bressler (European XFEL), Rainer Gehrke (DESY), Jan Grünert (European XFEL), Martin Müller (HZG), Uta Rütt (DESY), Christian Schroer (DESY), Edmund Welter (DESY) and Thomas White (DESY). 
Figure 1:

Participants of the annual European XFEL and DESY Photon Science Users' Meeting 2015.

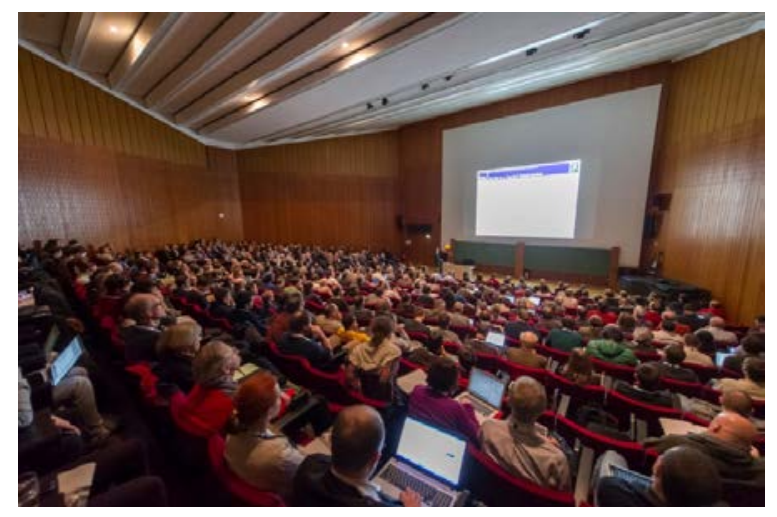

Figure 2:

Aerial view of the two FLASH FEL lines, PETRA III and the construction sites of the two PETRA extensions halls (dashed orange lines), and the CSSB building (dashed orange line), adjacent to the curved PETRA III experiment hall "Max von Laue", on the DESY campus in Hamburg in July 2014. The accelerator tunnel of the European XFEL starts here, while the experiment hall is situated in Schenefeld, about $3 \mathrm{~km}$ away.

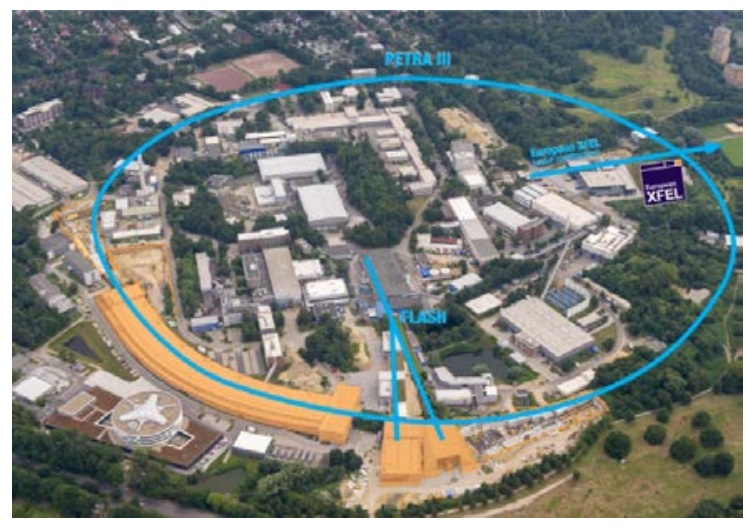

Figure 3:

Illustration draft of the European XFEL headquarters (XHQ) building in Schenefeld, that is located on top of the underground experiment hall. 


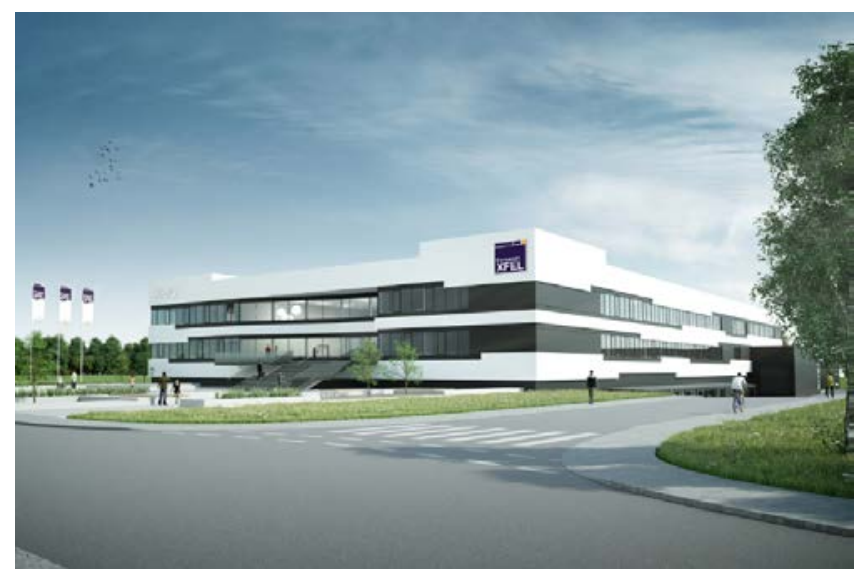

Figure 4:

European XFEL and DESY Photon Science Users' Meeting 2015: One of the two venues of the poster sessions, including a vendor exhibition.

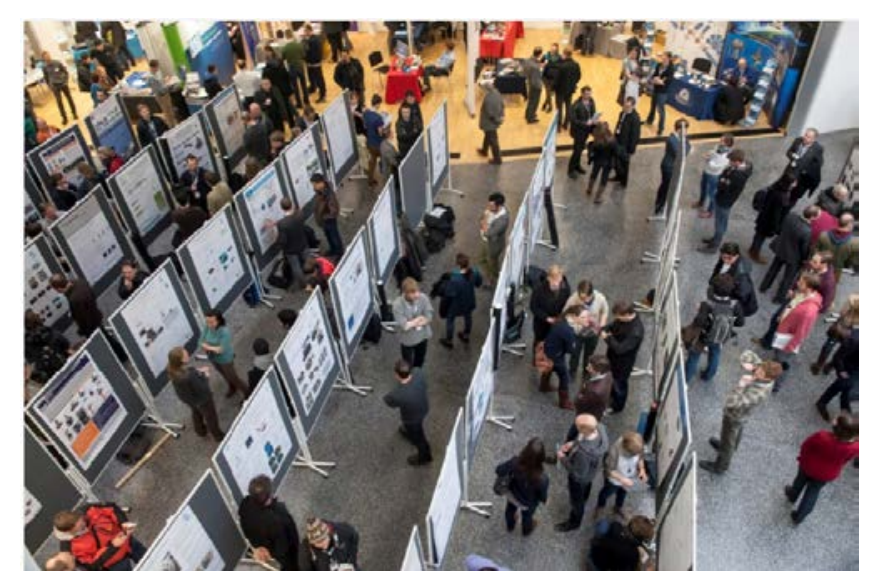

